\title{
Application of serum anti-Müllerian hormone levels in selecting patients with polycystic ovary syndrome for in vitro maturation treatment
}

\author{
Hyun Ha Seok ${ }^{1 *}$, Haengseok Song ${ }^{1,2 *}$, Sang Woo Lyu', You Shin Kim', Dong Ryul Lee ${ }^{1,2}$, Woo Sik Lee ', Tae Ki Yoon ${ }^{1}$ \\ 'Department of Obstetrics and Gynecology, Fertility Center of CHA Gangnam Medical Center, CHA University, Seoul; ${ }^{2}$ Department of Biomedical \\ Science, College of Life Science, CHA University, Seoul, Korea
}

Objective: The purpose of this study was to identify useful clinical factors for the identification of patients with polycystic ovary syndrome (PCOS) who would benefit from in vitro maturation (IVM) treatment without exhibiting compromised pregnancy outcomes.

Methods: A retrospective cohort study was performed of 186 consecutive patients with PCOS who underwent human chorionic gonadotropin-primed IVM treatment between March 2010 and March 2014. Only the first IVM cycle of each patient was included in this study. A retrospective case-control study was subsequently conducted to compare pregnancy outcomes between IVM and conventional in vitro fertilization (IVF) cycles.

Results: Through logistic regression analyses, we arrived at the novel finding that serum anti-Müllerian hormone (AMH) levels and the number of fertilized oocytes in IVM were independent predictive factors for live birth with unstandardized coefficients of 0.078 ( $95 \%$ confidence interval $[\mathrm{Cl}], 1.005-1.164 ; p=0.037)$ and $0.113(95 \% \mathrm{Cl}, 1.038-1.208 ; p=0.003)$, respectively. Furthermore, these two parameters were able to discriminate patients who experienced live births from non-pregnant IVM patients using cut-off levels of $8.5 \mathrm{ng} / \mathrm{mL}$ and five fertilized oocytes, respectively. A subsequent retrospective case-control study of patients with PCOS who had serum AMH levels $\geq 8.5 \mathrm{ng} / \mathrm{mL}$ showed that IVM had pregnancy outcomes comparable to conventional IVF, and that no cases of ovarian hyperstimulation syndrome were observed.

Conclusion: Serum AMH levels are a useful factor for predicting pregnancy outcomes in PCOS patients before the beginning of an IVM cycle. IVM may be an alternative to conventional IVF for PCOS patients if the patients are properly selected according to predictive factors such as serum AMH levels.

Keywords: Anti-Müllerian hormone; In vitro maturation of oocytes; Live-birth pregnancy rate; Polycystic ovary syndrome; Predictive factors

\section{Introduction}

Polycystic ovary syndrome (PCOS) is the most common fertility dis-

Received: Feb 4, 2016 · Revised: Feb 22, 2016 · Accepted: Feb 26, 2016 Corresponding author: Sang Woo Lyu

Department of Obstetrics and Gynecology, Fertility Center of CHA Gangnam Medical Center, CHA University, 566 Nonhyeon-ro, Gangnam-gu, Seoul 06135, Korea

Tel: +82-2-3468-3402 Fax:+82-2-3468-3464 E-mail: dung5038@cha.ac.kr

*These authors contributed equally to this work.

*This work was supported in part by the following National Research Foundation of Korea (NRF) grants funded by the Korean government (MEST): NRF2012R1A2A2A01011274 and NRF-2015R1A2A2A01006714.

This is an Open Access article distributed under the terms of the Creative Commons Attribution Non-Commercial License (http://creativecommons.org/licenses/by-nc/4.0/) which permits unrestricted non-commercial use, distribution, and reproduction in any medium, provided the original work is properly cited. order, affecting up to $20 \%$ of women attending fertility clinics [1]. One of the main characteristics of PCOS is chronic anovulation, with a significant proportion of affected women resistant to the induction of ovulation by clomiphene citrate [2]. Although gonadotropins can successfully induce ovulation in $75 \%$ of women who do not respond to clomiphene citrate, ovarian stimulation with exogenous gonadotropins significantly increases the risk of ovarian hyperstimulation syndrome (OHSS) in PCOS patients [3,4]. Several strategies have been developed to prevent OHSS, but none have proven completely successful $[5,6]$.

Since immature oocytes can be successfully matured and fertilized in vitro, resulting in the delivery of healthy babies, in vitro maturation (IVM) has emerged as an alternative to conventional in vitro fertilization (IVF) [7]. IVM involves the aspiration of immature oocytes from 
unstimulated or mildly stimulated ovaries, with subsequent maturation and fertilization in vitro. This is an attractive treatment modality since it reduces the cost of assisted reproductive technology (ART) and avoids the risk of OHSS [8]. These advantages may be more pronounced in women with PCOS due to their elevated risk of OHSS [9]. Furthermore, it is easy to identify multiple ovarian follicles by ultrasonography in PCOS patients, which makes it possible to retrieve a higher number of immature oocytes. This is consistent with previous reports indicating that the pregnancy rates of women with PCOS are higher than those of women with normal ovaries in IVM treatment, possibly due to the advantage of having a greater number of retrieved oocytes $[10,11]$. However, the pregnancy outcomes of IVM are still lower than those of conventional IVF, even in PCOS patients, which makes clinicians hesitant to choose IVM to treat PCOS patients when ART is needed [12]. Although a few studies have tried to identify independent factors predicting the pregnancy outcomes of IVM in PCOS patients, no clinical guidelines or protocols are available for identifying patients with PCOS who might be suitable for IVM without compromised pregnancy outcomes. Thus, we performed a retrospective cohort study to identify clinical factors that would be helpful in selecting a subgroup of patients with PCOS for possible IVM treatment, and found that serum anti-Müllerian hormone (AMH) levels are a useful factor in predicting the pregnancy outcomes of PCOS patients before beginning IVM treatment. Furthermore, a subsequent retrospective case-control study of patients with PCOS who had serum $\mathrm{AMH}$ levels $\geq 8.5 \mathrm{ng} / \mathrm{mL}$ reinforced this result. Our findings provide a clinical basis for establishing guidelines for selecting PCOS patients who would most likely benefit from IVM, with pregnancy outcomes comparable to those observed using IVF.

\section{Methods}

\section{Patients}

A retrospective cohort study was performed, including 186 consecutive patients with PCOS who underwent IVM treatment between March 2010 and March 2014. Only the first IVM cycle of each patient was included in this study. PCOS was diagnosed according to the Rotterdam PCOS consensus criteria. The exclusion criteria for all subjects were neoplastic, endocrinologic, or other gynecologic disorders capable of affecting pregnancy outcomes. This study was approved by the Institutional Review Board (reference number: GCl-14-18).

Based on the cut-off serum AMH level established in the retrospective cohort study, a retrospective case-control study was subsequently conducted to compare pregnancy outcomes between 107 IVM cycles and 177 conventional IVF cycles in PCOS patients. The conventional IVF cycles were conducted from March 2010 to March 2014. This case-control study was carried out by reviewing medical records, and written informed consent was waived. Cases and controls were defined as the first IVM and IVF cycles of patients with PCOS whose serum AMH levels were higher than $8.5 \mathrm{ng} / \mathrm{mL}$, respectively. All patients were matched for age, body mass index (BMI), infertility duration, and serum levels of follicle-stimulating hormone (FSH), and luteinizing hormone (LH).

\section{Hormone assessment}

Baseline hormone assays were performed using blood samples collected on day 2 or 3 of the menstrual cycle. In amenorrhoeic women, blood samples were collected after withdrawal bleeding induced by oral progestin (10 mg of Provera for 10 days, Pfizer Korea, Seoul, Korea). Basal serum levels of $\mathrm{AMH}, \mathrm{FSH}, \mathrm{LH}$, estradiol $\left(\mathrm{E}_{2}\right)$, thyroid stimulating hormone, prolactin, total testosterone, and dehydroepiandrosterone sulfate (DHEA-S) were measured. Serum AMH concentrations were measured using a two-site enzyme immunoassay (AMH/MIS ELISA kit, Immunotech, Marseille, France), with a sensitivity of 0.14 $\mathrm{ng} / \mathrm{mL}$, and intra- and interassay coefficients of variation of $12.3 \%$ and $14.2 \%$, respectively.

\section{Ultrasound measurements}

All subjects underwent a transvaginal ultrasound examination performed using a Voluson E8 (GE Healthcare, Buckinghamshire, UK). Sonographic data were obtained on day 2 or 3 after the onset of menstrual bleeding to check that no underlying gynecological disorders were present and to determine the antral follicle count (AFC). AFCs were measured in the rendered view after the ovary had been defined using virtual organ computer-aided analysis (VOCAL, GE Healthcare). A transvaginal ultrasound scan was repeated on cycle day 7 or 8 to exclude the development of a dominant follicle. Thereafter, it was performed to monitor the size of the follicle and the thickness of the endometrium.

\section{Oocyte collection and IVM cycle characteristics}

Immature oocytes were retrieved between 10 and 16 days of the cycle. When the endometrial thickness reached at least $6 \mathrm{~mm}$, patients received a subcutaneous injection of $250 \mu \mathrm{g}$ of human chorionic gonadotropin (hCG) (Ovidrel, Merck Serono, Darmstadt, Germany) and oocytes were collected 35 to 36 hours after the hCG injection. The immature oocytes that were retrieved were matured in vitro as previously described [13]. Briefly, transvaginal ultrasound-guided oocyte collection was performed using a 20-gauge single-lumen aspiration needle (Cook, Queensland, Australia), with a suction pressure of 10 to $12 \mathrm{kPa}$. Patients were sedated during oocyte collection with intravenous fentanyl (100 $\mu \mathrm{g}$, Daewon Pharmacology, Seoul, Korea) and midazolam (5 mg, Roche, Zurich, Switzerland).

The retrieved immature oocytes were isolated by washing the fol- 
licular aspirate in P1 medium (Irvine Scientific Co., Irvine, CA, USA) and cultured in organ culture dishes containing $800 \mu \mathrm{L}$ of IVM medium with $10 \mu \mathrm{M}$ melatonin at $37^{\circ} \mathrm{C}$ and $5 \% \mathrm{CO}_{2}$ in humidified air. After 24 to 48 hours, the oocytes were denuded of cumulus cells and their maturation was determined microscopically by the presence of the first polar body. The mature oocytes were fertilized using intracytoplasmic sperm injection (ICSI) in all cycles, and fertilization was assessed 16 to 18 hours after ICSI and defined as the appearance of two distinct pronuclei. These pronuclear zygotes were further cultured in $50 \mu \mathrm{L}$ of culture medium covered with oil (Quinn's Advantage Cleavage medium, Sage, Trumbull, CT, USA) for 48 hours until the intrauterine embryo transfer (ET). Embryo development was assessed on day 3 after insemination. A good-quality embryo was defined as having at least seven cells on day 3 , containing $<20 \%$ fragmentation, and exhibiting no apparent morphological abnormalities. A maximum of three embryos were transferred on day 3 after ICSI. Supernumerary embryos were cultured and frozen when they reached the blastocyst stage. For endometrial preparation, patients were given estradiol valerate (4 mg of Progynova orally, Schering, Berlin, Germany), starting on the day of oocyte retrieval. Luteal support was provided by transvaginal progesterone $(600 \mathrm{mg}$ of Utrogestan, Hanwha Pharmaceuticals, Seoul, Korea) starting from the day of fertilization. Serum $\beta$-hCG concentrations were measured 11 to 12 days after ET.

\section{Controlled ovarian stimulation for conventional IVF}

All patients underwent a gonadotropin-releasing hormone antagonist (Cetrotide, Merck Serono) protocol for controlled ovarian stimulation along with daily injections of recombinant FSH (Gonal-F, Merck Serono). A subcutaneous injection of $250 \mu \mathrm{g}$ of recombinant hCG (Ovidrel, Merck Serono) was administered to induce final follicular maturation when the mean diameter of at least two follicles reached $\geq 18 \mathrm{~mm}$ on transvaginal ultrasound. Luteal support was provided by transvaginal progesterone (600 mg of Utrogestan, Hanwha Pharmaceuticals) starting on the day of fertilization.

\section{Measurement of pregnancy outcomes}

Ongoing pregnancy was defined as the ultrasonographic visualization of a live fetus after 12 weeks of gestation. Live birth was defined as the delivery of a live infant after 28 weeks of gestation.

\section{Statistical analyses}

Statistical analyses were performed using the Statistical Package for the Social Science (IBM Co., Armonk, NY, USA). Comparisons of numerical variables were performed using the Student's $t$-test. Categorical variables were compared using the chi-square test. Logistic regression analysis was performed to identify independent risk factors and to predict the probability of live birth. Receiver operating characteristic (ROC) curve analyses were conducted for the serum AMH level and the number of fertilized oocytes in IVM to distinguish patients who experienced live births from non-pregnant patients in IVM cycles. In order to measure the linear correlation between candidatepredictive parameters and the number of fertilized oocytes in IVM, Pearson's correlation coefficient and the corresponding $p$-values were calculated through correlation analysis. In order to predict the number of fertilized oocytes using these variables, multivariate linear regression analysis was conducted following a stepwise regression method. The $p$-values $<0.05$ were defined as indicating statistical significance.

\section{Results}

This study included 186 consecutive patients with PCOS who underwent hCG-primed IVM treatment without gonadotropin stimula-

Table 1. Characteristics and basal hormone concentrations of patients with PCOS who underwent IVM

\begin{tabular}{|c|c|c|c|c|}
\hline & All patients $(n=186)$ & Live birth $(n=56)$ & No live birth $(n=130)$ & $p$-value \\
\hline Age (yr) & $32.33 \pm 3.22$ & $31.95 \pm 3.00$ & $32.50 \pm 3.31$ & 0.290 \\
\hline $\operatorname{BMl}\left(\mathrm{kg} / \mathrm{m}^{2}\right)$ & $22.06 \pm 3.35$ & $21.68 \pm 3.16$ & $22.23 \pm 3.43$ & 0.310 \\
\hline Duration of infertility (yr) & $3.88 \pm 2.55$ & $3.89 \pm 2.72$ & $3.88 \pm 2.49$ & 0.987 \\
\hline Antral follicle count & $19.66 \pm 5.89$ & $21.04 \pm 7.36$ & $19.06 \pm 5.05$ & 0.071 \\
\hline $\mathrm{AMH}(\mathrm{ng} / \mathrm{mL})$ & $10.30 \pm 4.91$ & $12.29 \pm 5.94$ & $9.44 \pm 4.13$ & 0.002 \\
\hline FSH (IU/L) & $7.26 \pm 2.01$ & $7.55 \pm 2.10$ & $7.14 \pm 1.97$ & 0.210 \\
\hline $\mathrm{LH}(\mathrm{IU} / \mathrm{L})$ & $9.22 \pm 5.54$ & $10.67 \pm 6.21$ & $8.62 \pm 5.15$ & 0.025 \\
\hline Testosterone $(\mathrm{ng} / \mathrm{mL})$ & $0.46 \pm 0.18$ & $0.42 \pm 0.18$ & $0.41 \pm 0.18$ & 0.144 \\
\hline DHEA-S $(\mu \mathrm{g} / \mathrm{dL})$ & $181.78 \pm 72.58$ & $189.18 \pm 72.25$ & $178.54 \pm 72.79$ & 0.379 \\
\hline Endometrial thickness on ET (mm) & $6.5 \pm 1.23$ & $6.72 \pm 1.34$ & $6.41 \pm 1.17$ & 0.155 \\
\hline
\end{tabular}

Values are presented as mean \pm standard deviation.

PCOS, polycystic ovary syndrome; IVM, in vitro maturation; BMI, body mass index; AMH, anti-Müllerian hormone; FSH, follicle-stimulating hormone; LH, luteinizing hormone; DHEA-S, dehydroepiandrosterone sulfate; ET, embryo transfer. 
tion. The mean age of the patients was $32.33 \pm 3.22$ years, the mean BMI was $22.06 \pm 3.35 \mathrm{~kg} / \mathrm{m}^{2}$, the duration of infertility was $3.88 \pm 2.55$ years, the mean number of immature oocytes retrieved was $16.15 \pm 8.43$, and the overall live birth rate was $30.1 \%(56 / 186)$.

Comparisons of patients' characteristics (age, BMI, and AFCs) and basal hormone concentrations (AMH, FSH, LH, total testosterone, and DHEA-S) between the live birth and non-pregnant groups showed that serum levels of AMH and LH were significantly higher in the patients who experienced live birth $(\mathrm{AMH}, 12.29 \pm 5.94 \mathrm{ng} / \mathrm{mL}$ vs. $9.44 \pm 4.13 \mathrm{ng} / \mathrm{mL}, p=0.002 ; \mathrm{LH}, 10.67 \pm 6.21 \mathrm{IU} / \mathrm{L}$ vs. $8.62 \pm 5.15 \mathrm{IU} / \mathrm{L}$, $p=0.025$ ) (Table 1).

Among the laboratory outcomes (Table 2), the numbers of immature oocytes retrieved, matured oocytes in IVM, fertilized oocytes, and frozen embryos were significantly higher in the patients who experienced live birth $(18.93 \pm 10.04$ vs. $14.95 \pm 7.35, p=0.009$; $11.21 \pm 6.40$ vs. $8.03 \pm 4.31, p=0.001 ; 9.59 \pm 5.86$ vs. $6.56 \pm 3.63$, $p=0.001 ; 0.80 \pm 1.28$ vs. $0.29 \pm 0.83, p=0.007$, respectively).

Logistic regression analysis indicated that among all the parameters listed in Tables 1 and 2, serum AMH level and the number of fertilized oocytes in IVM were independent predictive factors for live birth with unstandardized coefficients (95\% confidence interval [CI]) of $0.078(95 \% \mathrm{Cl}, 1.005-1.164 ; p=0.037)$ and $0.113(95 \% \mathrm{Cl}, 1.038-$ $1.208, p=0.003)$, respectively. Furthermore, ROC curve analysis showed that these two parameters were capable of distinguishing patients who experienced live birth from non-pregnant patients in IVM treatment (AMH: area under the curve $[A U C]=0.640,95 \% \mathrm{Cl}$,
0.551-0.730, $p=0.002$; number of fertilized oocytes: $\mathrm{AUC}=0.667$, $95 \% \mathrm{Cl}, 0.581-0.752, p<0.001)$. The cut-off levels for the serum AMH level and the number of fertilized oocytes were determined to be 8.5 $\mathrm{ng} / \mathrm{mL}$ and five oocytes, respectively. Patients with values greater than these cut-off levels had significantly higher live birth rates (36.4\% [39/107] vs. $21.5 \%$ [17/79], odds ratio $=2.092, p=0.028$; $35.0 \%[49 / 140]$ vs. $15.2 \%[7 / 46]$, odds ratio $=3.000, p=0.011$, respectively) (Table 3).

We then performed correlation analyses and multivariate linear regression to examine whether any factors pre dicted the number of fertilized oocytes before the initiation of IVM cycles. Serum AMH levels and AFCs exhibited a linear correlation with the number of fertilized oocytes in IVM. Subsequently, multivariate linear regression analysis using the stepwise regression method showed that the unstandardized coefficients were $0.227(p<0.001)$ and $0.187(p=0.007)$ for AFCs and serum AMH levels, respectively. Collectively, these results suggest that a patient's AFC and serum AMH level can be used to estimate the number of fertilized oocytes, one of the predictive factors for pregnancy outcomes in the IVM treatment of PCOS patients (number of estimated fertilized oocytes $=1.243+[0.227 \times$ AFC $]$ $+[0.187 \times \mathrm{AMH}])$.

In order to validate the clinical significance of serum AMH levels in identifying the patients with PCOS most likely to benefit from IVM without compromised pregnancy outcomes, we subsequently compared the pregnancy outcomes of the patients in the IVM cohort $(n=107)$ with those of patients with PCOS who underwent conven-

Table 2. Laboratory outcomes of patients with PCOS who underwent IVM

\begin{tabular}{|c|c|c|c|c|}
\hline & All patients $(n=186)$ & Live birth $(n=56)$ & No live birth $(n=130)$ & $p$-value \\
\hline Immature oocytes retrieved (n) & $16.15 \pm 8.43$ & $18.93 \pm 10.04$ & $14.95 \pm 7.35$ & 0.009 \\
\hline Matured oocytes (n) & $8.99 \pm 5.22$ & $11.21 \pm 6.40$ & $8.03 \pm 4.31$ & 0.001 \\
\hline Fertilized 2PN oocytes (n) & $7.47 \pm 4.62$ & $9.59 \pm 5.86$ & $6.56 \pm 3.63$ & 0.001 \\
\hline Maturation rate & $55.6(1,671 / 3,004)$ & $59.2(628 / 1,060)$ & $53.7(1,044 / 1,944)$ & 0.003 \\
\hline Fertilization rate & $83.2(1,390 / 1,671)$ & $85.5(537 / 628)$ & $81.7(853 / 1,044)$ & 0.044 \\
\hline Transferred embryos (n) & $2.44 \pm 0.52$ & $2.38 \pm 0.48$ & $2.46 \pm 0.54$ & 0.287 \\
\hline Frozen embryos (n) & $0.45 \pm 1.02$ & $0.80 \pm 1.28$ & $0.29 \pm 0.83$ & 0.007 \\
\hline
\end{tabular}

Values are presented as mean \pm standard deviation or percent (number). PCOS, polycystic ovary syndrome; IVM, in vitro maturation; PN, pronuclei.

Table 3. Live birth rate by cut-off value of serum AMH and number of fertilized oocytes in patients with PCOS who underwent IVM

\begin{tabular}{|c|c|c|c|c|c|}
\hline & \multirow{2}{*}{ Overall } & \multicolumn{2}{|c|}{ AMH levels } & \multicolumn{2}{|c|}{ No. of fertilized oocytes } \\
\hline & & $<8.5 \mathrm{ng} / \mathrm{mL}$ & $\geq 8.5 \mathrm{ng} / \mathrm{mL}$ & $<5$ & $\geq 5$ \\
\hline Live birth rate $(\%, \mathrm{n})$ & $30.1(56 / 186)$ & $21.5(17 / 79)$ & $36.4(39 / 107)$ & $15.2(7 / 46)$ & $35.0(49 / 140)$ \\
\hline$p$-value & & 0.028 & & 0.011 & \\
\hline Odds ratio & & 2.092 & & 3 & \\
\hline 95\% Confidence interval & & $1.075-4.069$ & & $1.249-7.206$ & \\
\hline
\end{tabular}

AMH, anti-Müllerian hormone; PCOS, polycystic ovary syndrome; IVM, in vitro maturation. 
Table 4. Pregnancy outcomes of IVM and conventional IVF in PCOS patients with serum AMH levels $\geq 8.5 \mathrm{ng} / \mathrm{mL}$

\begin{tabular}{lccc}
\hline & IVM $(n=107)$ & Conventional IVF $(n=177)$ & $p$-value \\
\hline Pregnancy rate per OPU & $46.7(50 / 107)$ & $40.1(71 / 177)$ & 0.275 \\
Pregnancy rate per ET & $46.7(50 / 107)$ & $57.7(71 / 123)$ & 0.096 \\
Ongoing pregnancy rate per OPU & $37.4(40 / 107)$ & $30.5(54 / 177)$ & 0.233 \\
Ongoing pregnancy rate per ET & $37.4(40 / 107)$ & $43.9(54 / 123)$ & 0.316 \\
Live birth rate per OPU & $36.4(39 / 107)$ & $29.4(52 / 177)$ & 0.216 \\
Live birth rate per ET & $36.4(39 / 107)$ & $42.3(52 / 123)$ & 0.367 \\
Miscarriage rate & $10.0(5 / 50)$ & $11.3(11 / 71)$ & 0.321 \\
OHSS & $0(0 / 107)$ & $21.5(38 / 177)$ & $<0.001$ \\
Embryo all freezing & $0(0 / 107)$ & $30.5(54 / 177)$ & $<0.001$ \\
\hline
\end{tabular}

Values are presented as percent (number).

IVM, in vitro maturation; IVF, in vitro fertilization; PCOS, polycystic ovary syndrome; AMH, anti-Müllerian hormone; OPU, ovum pick-up; ET, embryo transfer; OHSS, ovarian hyperstimulation syndrome.

tional IVF cycles $(\mathrm{n}=177)$ as a retrospective case-control study. No differences were found in terms of age, BMI, infertility duration and serum levels of FSH and LH (data not shown). IVM cycles showed comparable pregnancy, ongoing pregnancy, and live birth outcomes per ET (pregnancy rate, $46.7 \%$ [50/107] vs. $57.7 \%$ [71/123], $p=0.096$; ongoing pregnancy rate, $37.4 \%$ [40/107] vs. $43.9 \%$ [54/123], $p=0.316$; live birth rate, $36.4 \%[39 / 107]$ vs. $42.3 \%[52 / 123], p=0.367$ ); and no cases of OHSS were observed in the patients who received IVM treatment (0\% [0/107] vs. $21.5 \%$ [38/177], $p<0.001)$ (Table 4).

\section{Discussion}

IVM is an attractive ART option for patients with PCOS or those with polycystic-like ovaries because it eliminates the risk of OHSS that is present during conventional IVF. However, it is difficult for clinicians to choose IVM as an alternative for PCOS because the pregnancy outcomes of IVM are poorer than those of conventional IVF. Thus, it is clinically critical to identify predictive factors capable of distinguishing patients with PCOS who could benefit from IVM without compromised pregnancy outcomes. Previous studies have suggested that the number of immature oocytes retrieved may be positively correlated with the pregnancy outcomes of patients undergoing IVM $[10,11,14-19]$. However, it is not known whether any aspects of the hormonal and clinical profiles of patients could predict pregnancy outcomes before the initiation of IVM treatment. Herein, we have identified serum AMH levels as a valuable predictive factor that can be used to identify patients who are suitable for IVM. Furthermore, the subsequent retrospective case-control analysis of patients with PCOS whose serum AMH levels were higher than $8.5 \mathrm{ng} / \mathrm{mL}$ reinforced the finding that IVM led to pregnancy outcomes that are comparable to conventional IVF in these patients (Table 4). Our results suggest that IVM is a viable alternative ART treatment for PCOS patients if the patients are properly selected.
It is well known that serum AMH level is the best currently available measure of the ovarian reserve. Not only is AMH used in a variety of clinical situations, such as infertility treatment including ART, it also is used for the prediction of reproductive lifespan, ovarian dysfunction (especially PCOS), and gonadotoxic cancer therapy or ovarian surgery [20]. However, only a handful of studies have evaluated the clinical significance of serum AMH levels in IVM treatment $[15,18,19]$. These studies have suggested that serum AMH levels are associated with the number of immature oocytes retrieved or the number of matured oocytes in IVM. Although the oocyte yield may be associated with pregnancy outcomes, it is impossible to determine the exact number of oocytes that will be produced before the cycle starts. Thus, these previous studies were not able to show any direct relevance of serum AMH levels to pregnancy outcomes. They were practically limited because the enrolled patients were heterogeneous and/or the pregnancy outcomes were too poor to evaluate the significance of serum AMH levels in patients undergoing IVM. Furthermore, they did not include the live birth rate as a pregnancy outcome. Our results, for the first time, demonstrated that serum $\mathrm{AMH}$ levels $\geq 8.5 \mathrm{ng} / \mathrm{mL}$ were an independent factor capable of directly predicting pregnancy outcomes in patients with $\mathrm{PCOS}$ before the initiation of IVM treatment.

OHSS is a serious iatrogenic complication observed in some patients who undergo gonadotropic ovarian stimulation. In severe forms of OHSS, life-threatening complications may occur, including pleural or pericardial effusion, adult respiratory distress syndrome, thromboembolic events, myocardial or cerebral infarctions, and even death [21]. Various techniques exist for preventing OHSS, but none are completely effective. PCOS is a well-known pre-existing risk factor for OHSS, and serum AMH levels have been associated with the incidence of OHSS in IVF cycles [22-24]. The present study showed that no patients experienced OHSS during IVM, as was expected since exogenous ovarian stimulation drugs were not used (Table 4). 
Collectively, these results indicate that patients with PCOS who are at a high risk for OHSS in conventional IVF cycles would be good candidates for IVM without any concerns regarding OHSS.

The group of patients with PCOS whose AMH levels were greater than $8.5 \mathrm{ng} / \mathrm{mL}$ had significantly higher pregnancy and live birth rates (Table 3 ). This could be explained by the observation that patients in this group were more likely to produce a larger number of good quality embryos. In fact, the number of immature oocytes retrieved, in vitro-matured oocytes, fertilized oocytes, and frozen embryos were significantly higher in the patients who experienced live birth (Table 2). Patients with serum AMH levels higher than $8.5 \mathrm{ng} / \mathrm{mL}$ had a greater number of immature oocytes retrieved and subsequently matured oocytes (data not shown), leading to more good-quality embryos and remaining frozen blastocysts, respectively $(1.64 \pm 1.15$ vs. $2.20 \pm 1.56, p=0.01 ; 0.24 \pm 0.63$ vs. $0.59 \pm 1.20, p=0.01$ )

IVM has been shown to be less successful than conventional IVF $[10,12,25,26]$ or comparable pregnancy outcomes [27]. Furthermore, two recent studies have reported that the live birth rates of patients undergoing IVM were significantly lower than those of patients undergoing conventional IVF $[8,28]$. However, we present evidence that IVM may have comparable live birth rates and ongoing pregnancy rates if patients with PCOS are carefully selected (Table 4). Since the best way to treat PCOS in ART is to maximize pregnancy outcomes while minimizing the risk of OHSS, IVM is a safe and efficient alternative ART treatment if the patients are properly selected based on robust predictive factors, such as serum AMH levels. Although this was a retrospective study, we attempted to compensate for the practical limitations inherent to retrospective studies. Only the first cycles of IVM and conventional IVF in PCOS patients were included, and the largest cohort of patients with PCOS yet analyzed was included in this study. Nonetheless, this study underscores the relevance of welldesigned randomized prospective studies in demonstrating the clinical significance of serum AMH levels as a guideline for choosing ART options for PCOS patients.

In conclusion, serum AMH levels are a useful factor in predicting pregnancy outcomes for patients with PCOS before beginning IVM treatment. This study suggests that IVM may be an attractive ART option for PCOS patients if the patients are properly selected using robust predictive factors, such as serum AMH levels.

\section{Conflict of interest}

No potential conflict of interest relevant to this article was reported.

\section{References}

1. Diamanti-Kandarakis E, Kouli C, Tsianateli T, Bergiele A. Thera- peutic effects of metformin on insulin resistance and hyperandrogenism in polycystic ovary syndrome. Eur J Endocrinol 1998; 138:269-74.

2. Chian RC. In-vitro maturation of immature oocytes for infertile women with PCOS. Reprod Biomed Online 2004;8:547-52.

3. Smith V, Osianlis T, Vollenhoven B. Prevention of ovarian hyperstimulation syndrome: a review. Obstet Gynecol Int 2015;2015: 514159.

4. Nastri CO, Teixeira DM, Moroni RM, Leitao VM, Martins WP. Ovarian hyperstimulation syndrome: pathophysiology, staging, prediction and prevention. Ultrasound Obstet Gynecol 2015;45: 377-93.

5. Nastri CO, Ferriani RA, Rocha IA, Martins WP. Ovarian hyperstimulation syndrome: pathophysiology and prevention. J Assist Reprod Genet 2010;27:121-8.

6. Tiitinen A, Husa LM, Tulppala M, Simberg N, Seppala M. The effect of cryopreservation in prevention of ovarian hyperstimulation syndrome. Br J Obstet Gynaecol 1995;102:326-9.

7. Cha KY, Koo JJ, Ko J, Choi DH, Han SY, Yoon TK. Pregnancy after in vitro fertilization of human follicular oocytes collected from nonstimulated cycles, their culture in vitro and their transfer in a donor oocyte program. Fertil Steril 1991;55:109-13.

8. Das M, Son WY, Buckett W, Tulandi T, Holzer H. In-vitro maturation versus IVF with $\mathrm{GnRH}$ antagonist for women with polycystic ovary syndrome: treatment outcome and rates of ovarian hyperstimulation syndrome. Reprod Biomed Online 2014;29:545-51.

9. Shalom-Paz E, Holzer H, Son W, Levin I, Tan SL, Almog B. PCOS patients can benefit from in vitro maturation (IVM) of oocytes. Eur J Obstet Gynecol Reprod Biol 2012;165:53-6.

10. Child TJ, Abdul-Jalil AK, Gulekli B, Tan SL. In vitro maturation and fertilization of oocytes from unstimulated normal ovaries, polycystic ovaries, and women with polycystic ovary syndrome. Fertil Steril 2001;76:936-42.

11. Child TJ, Sylvestre C, Pirwany I, Tan SL. Basal serum levels of FSH and estradiol in ovulatory and anovulatory women undergoing treatment by in-vitro maturation of immature oocytes. Hum Reprod 2002;17:1997-2002.

12. Gremeau AS, Andreadis N, Fatum M, Craig J, Turner K, McVeigh E, et al. In vitro maturation or in vitro fertilization for women with polycystic ovaries? A case-control study of 194 treatment cycles. Fertil Steril 2012;98:355-60.

13. Chang EM, Han JE, Seok HH, Lee DR, Yoon TK, Lee WS. Insulin resistance does not affect early embryo development but lowers implantation rate in in vitro maturation-in vitro fertilization-embryo transfer cycle. Clin Endocrinol (Oxf) 2013;79:93-9.

14. Mikkelsen AL, Andersson AM, Skakkebaek NE, Lindenberg S. Basal concentrations of oestradiol may predict the outcome of 
in-vitro maturation in regularly menstruating women. Hum Reprod 2001;16:862-7.

15. Fadini R, Comi R, Mignini Renzini M, Coticchio G, Crippa M, De Ponti $\mathrm{E}$, et al. Anti-mullerian hormone as a predictive marker for the selection of women for oocyte in vitro maturation treatment. J Assist Reprod Genet 2011;28:501-8.

16. Child TJ, Phillips SJ, Abdul-Jalil AK, Gulekli B, Tan SL. A comparison of in vitro maturation and in vitro fertilization for women with polycystic ovaries. Obstet Gynecol 2002;100:665-70.

17. Tan SL, Child TJ. In-vitro maturation of oocytes from unstimulated polycystic ovaries. Reprod Biomed Online 2002;4 Suppl 1:1823.

18. Guzman L, Ortega-Hrepich C, Polyzos NP, Anckaert E, Verheyen G, Coucke W, et al. A prediction model to select PCOS patients suitable for IVM treatment based on anti-Mullerian hormone and antral follicle count. Hum Reprod 2013;28:1261-6.

19. Kedem A, Yerushalmi GM, Maman E, Hemi R, Hanochi M, Hourvitz $A$. What is the optimal threshold of serum Anti-Mullerian hormone (AMH) necessary for IVM treatments? J Assist Reprod Genet 2013;30:745-51.

20. Broer SL, Broekmans FJ, Laven JS, Fauser BC. Anti-Mullerian hormone: ovarian reserve testing and its potential clinical implications. Hum Reprod Update 2014;20:688-701.

21. Delvigne A, Rozenberg S. Review of clinical course and treatment of ovarian hyperstimulation syndrome (OHSS). Hum Reprod Update 2003;9:77-96.

22. Ocal P, Sahmay S, Cetin M, Irez T, Guralp O, Cepni I. Serum antiMullerian hormone and antral follicle count as predictive mark- ers of OHSS in ART cycles. J Assist Reprod Genet 2011;28:1197203.

23. Nakhuda GS, Chu MC, Wang JG, Sauer MV, Lobo RA. Elevated serum mullerian-inhibiting substance may be a marker for ovarian hyperstimulation syndrome in normal women undergoing in vitro fertilization. Fertil Steril 2006;85:1541-3.

24. Tal R, Seifer DB, Khanimov M, Malter HE, Grazi RV, Leader B. Characterization of women with elevated antimullerian hormone levels (AMH): correlation of AMH with polycystic ovarian syndrome phenotypes and assisted reproductive technology outcomes. Am J Obstet Gynecol 2014;211:59.e1-8.

25. Cha KY, Chung HM, Lee DR, Kwon H, Chung MK, Park LS, et al. Obstetric outcome of patients with polycystic ovary syndrome treated by in vitro maturation and in vitro fertilization-embryo transfer. Fertil Steril 2005;83:1461-5.

26. Lin YH, Hwang JL, Huang LW, Mu SC, Seow KM, Chung J, et al. Combination of FSH priming and hCG priming for in-vitro maturation of human oocytes. Hum Reprod 2003;18:1632-6.

27. Shavit T, Ellenbogen A, Michaeli M, Kartchovsky E, Ruzov O, Shalom-Paz E. In-vitro maturation of oocytes vs in-vitro fertilization with a gonadotropin-releasing hormone antagonist for women with polycystic ovarian syndrome: can superiority be defined? Eur J Obstet Gynecol Reprod Biol 2014;179:46-50.

28. Walls ML, Hunter T, Ryan JP, Keelan JA, Nathan E, Hart RJ. In vitro maturation as an alternative to standard in vitro fertilization for patients diagnosed with polycystic ovaries: a comparative analysis of fresh, frozen and cumulative cycle outcomes. Hum Reprod 2015;30:88-96. 